\title{
Untersuchungen zur Immunitätslage der impfpräventablen Kinderkrankheiten Röteln, Masern, Mumps und Windpocken
}

\author{
S. Buxbaum, H. W. Doerr, R. Allwinn \\ Institut für Medizinische Virologie der Universitätsklinik Frankfurt/Main
}

Grundproblematilk und Fragestellung: Infektionen mit Masern, Mumps, Röteln und Varizellen gelten als typische Kinderkrankheiten. In der Regel heilen sie komplikationslos aus und hinterlassen eine lebenslange Immunität. Jedoch gibt es durchaus schwere Krankheitsverläufe, besonders auch bei Immungeschwächten. In der Frühschwangerschaft sind zudem Infektionen mit Röteln aufgrund der Embryopathie sehr gefürchtet. Die Impfung gegen Masern, Mumps und Röteln wird seit den 80er Jahren öffentlich empfohlen, seit Juli 2001 ebenfalls die Varizellenimpfung bei nicht-immunen Jugendlichen. In dieser Studie wurden die Populationsimmunität und das Vorhandensein von Immunitätslücken gegenüber diesen Erkrankungen untersucht. Methodilk: Zur Bestimmung der Immunität wurden Serumproben ausgewertet, die am Institut für Medizinische Virologie der Universitätsklinik Frankfurt zwischen 1.1.1999 und 31.12.2000 auf Antikörper gegen Masern ( $n=915)$, Mumps ( $n=857)$, Röteln $(n=1886)$ und Varizellen $(n=2291)$ untersucht wurden. Die Bestimmung der Seroprävalenzen erfolgte in verschiedenen Altersklassen.

Ergebnisse: Es ließ sich gegenüber Masern, Mumps, Röteln und Varizellen Immunitätslücken finden, die im Kindesalter am ausgeprägtesten, mit zunehmenden Lebensalter jedoch rückläufig waren. Bei Varizellen fiel der Anteil der Seronegativen in der Altersgruppe der 1 - bis 4 -Jährigen von $74 \%$ auf $32 \%$ bei den 5 - bis 9-Jährigen. Auch bei Röteln war die Anzahl der Seronegativen ebenfalls im Kindesalter mit $40 \%$ am höchsten und sank auf weniger als $10 \%$ in der Altersklasse der 15 - bis 19 -Jährigen ab. Von dieser Altersklasse an zeigten etwa $80 \%$ der Seropositiven eine ausreichende Rötelnimmunität.

Folgerungen: Die vorliegende Studie zeigt, dass weiterhin Immunitätslücken bestehen. Dies gilt besonders auch gegenüber Varizellen; hier ist neuerdings eine Impfung der seronegativen Jugendlichen empfohlen. Prinzipiell sollten Immunisierungsraten von über $95 \%$ gerade in den gefährdeten Jahrgängen des frühen Kindesalters erreicht werden.
Epidemiological analysis of immunity against vaccine-preventable diseases: rubella, measles, mumps and varicella zoster virus

Background and objective: Measles, mumps, rubella and varicella zoster virus (VZV) infections are regarded as typical diseases of childhood: They are normally clinically mild and result in lifelong immunity. Severe clinical disease is known in immunocompromised patients; rubella virus infections during pregnancy often result in congenital rubella syndrome. All these diseases are preventable by vaccination which is recommended in Germany, recently vaccination against VZV for teenager without immunity since July 2001. In the following study we screened for immunity against the four viruses.

Patients and methods: Serum samples were obtained at the Institute of Medical Virology Frankfurt/Main from January 1999 until December 2000 . We tested for specific antibodies against measles $(n=915)$, against mumps $(n=857)$, against rubella $(n=1886)$ and against VZV $(n=2291)$. Seroprevalences were determined in different age groups.

Results: Altogether the highest rate of seronegatives is detected in younger children. VZV-seronegativity rates decrease from $74 \%$ to $32 \%$ in younger children. Against rubella also in this age group rate of seronegatives is found in $40 \%$ and less than $10 \%$ by teenagers. From this age group also immunity against rubella is found approximately in $80 \%$ of seropositives.

Conclusions: The following study shows that high seronegative rates are detectable, and here specially against VZV-specific antibodies. For seronegative teenagers, vaccination against VZV is now recommended in Germany. Immunization rates of at least $95 \%$ in childhood would be effective in avoiding severe courses of disease and giving protection in pregnancy.
Masern, Mumps, Röteln und Windpocken sind klassische Viruskrankheiten der Kindheit, die trotz durchschnittlich guter Prognose jedoch auch wegen bestimmter Komplikationen (Neurotropismus, vertikale Rötelntransmission) gefürchtet sind und damit auch eine erhebliche Belastung des Gesundheitssystems bedeuten können (15). Gegen Masern, Mumps und Röteln wird daher die zweimalige simultane Populationsimpfung mit attenuierten Virusstämmen bereits im Kleinkindesalter von der Ständigen Impfkommission (STIKO) am Robert Koch Institut in Berlin öffentlich empfohlen. Verschiedene mathematische Modellrechnungen haben gezeigt, dass eine solche Impfung - z. B. gegen Masern - nur wirksam ist, wenn möglichst über 85\% der Kinder durchimmunisiert sind $(1,9)$. Anderenfalls kann die Gefahr der Infektionen sogar zunehmen, weil die Wildvirusdurchseuchung nicht unterbun-

Dtsch Med Wochenschr 2001; 126: 1289-1293

(c) Georg Thieme Verlag Stuttgart · New York den, sondern nur verlangsamt wird. Das hat zur Folge, dass auch ältere Jugendliche oder sogar Erwachsene infiziert werden, bei welchen Komplikationen, insbesondere zentralnervöser Art häufiger registriert werden. Auch das Risiko der gebärfähigen Frauen, sich mit dem teratogenen Rötelnvirus zu infizieren, nimmt dann zu (3).

Abgeheilte Windpocken hinterlassen das Virus latent in den Spinalganglien am Rückenmark. Wenn nach Jahrzehnten die (zelluläre) Immunität abgenommen hat, kann eine Infektreaktivierung in Form eines Herpes Zoster auftreten. Dies ist eine schmerzhafte dermatologische und neurologische Erkrankung des Alters. Pro- und retrospektive Studien haben gezeigt, dass ca. $20 \%$ aller Menschen über 60 Jahre bereits einen Herpes Zoster durchgemacht haben, der in etlichen Fällen als äußerst unangenehme postzosterische Neuralgie lebenslang bestehen bleiben kann (11). Auch in der Kindheit und im jungen Erwachsenenalter besteht die Gefahr eines Herpes 


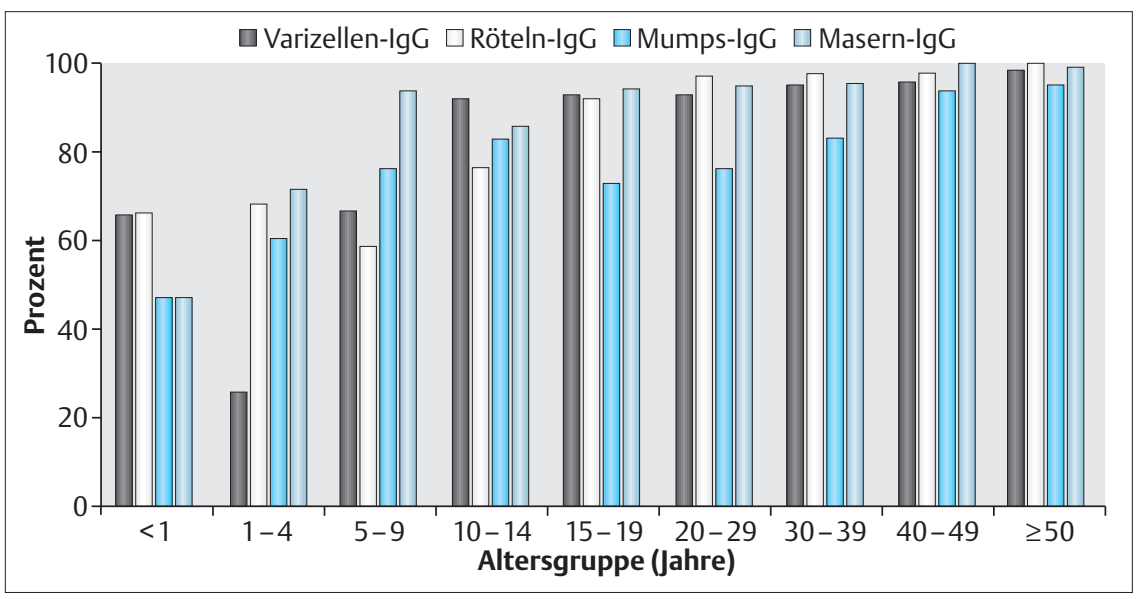

Abb.1 Prävalenz der lgG-Antikörper gegen Masern, Mumps, Röteln und Varizellen in verschiedenen Altersklassen.

Zoster, wenn ein zellulärer Immundefekt auftritt. So ist der Herpes Zoster auch eine Markerkrankheit von AIDS. Sehr gefürchtet sind Windpocken und Zosterausbrüche auf Leukämiestationen. Seit einigen Jahren wird daher gefordert, auch gegen die Infektion mit dem Windpocken (Varicella-) ZosterVirus (VZV) eine generelle Impfung mit einem attenuierten Virusstamm, am besten in Kombination mit der MMR-Vakzination, einzuführen. Aktuell wird die Varizellen-Impfung für nicht immune Jugendliche empfohlen (13).

Im Folgenden legen wir eine retrospektive Analyse der Populationsimmunität gegen Masern, Mumps, Röteln und Windpocken auf der Basis laufender labordiagnostischer Untersuchungen vor. Diese werden im Institut für Medizinische Virologie der Universität Frankfurt routinemäßig durchgeführt, um noch vorhandene Immunschutzlücken in der Bevölkerung aufzudecken. Solche Untersuchungen haben sich bereits im WHO-Programm der Poliomyelitisimmunität bewährt $(5,6,10)$.

\section{Material und Methoden}

Ausgewertet wurden Serumproben, die zwischen dem 1. Januar 1999 und dem 31. Dezember 2000 im Institut für Medizinische Virologie (akkreditiert nach DIN EN 45001) der Universitätsklinik Frankfurt/Main zur Abklärung des Immunstatus auf Antikörper gegen Röteln (hier auch mittels Hämagglutinationshemmtest, HHT), Masern, Mumps und Varizellen untersucht wurden. Mittels kommerzieller Testkits (Enzygnost, Fa. Behring) erfolgte die Bestimmung spezifischer IgG-Antikörper (in IE; "Internationale Einheiten"). Ausgeschlossen wurden IgMpositive Seren. Eine Untersuchung auf spezifische IgA-Antikörper erfolgte bei $77 \%$ der auf Varizellen getesteten Seren. Alle Proben stammten von Patienten, die entweder in der Uniklinik Frankfurt selbst oder in Kliniken, bzw. Praxen aus dem Einzugsgebiet Rhein/Main behandelt, bzw. betreut wurden.

Zur Auswertung kamen 915 Seren zur Masern-, 857 Seren zur Mumps- und 1886 Seren zur Röteln-Antikörperbestimmung. 2291 Seren wurden auf Antikörper gegen Varizellen untersucht. Alle Auswertungen wurden aus Erstuntersuchungen erhalten.

Die in der Studie bestimmte Immunität unterscheidet nicht die natürliche, durch Wildvirusinfektion erworbene von der durch eine Impfung erzielten Immunität. Während der Anteil männlicher und weiblicher Patienten bei den Untersuchungen auf Masern, Mumps und Varizellen vergleichbar hoch ist, ver- schiebt er sich bei den Röteln-Immunitätsuntersuchungen in den Altersgruppen von 15-49 Jahren deutlich zugunsten der Patientinnen; dies erklärt sich durch die Fragestellung der Röteln-Immunität bei gebärfähigen Frauen im Hinblick auf das gefürchtete kongenitale Rötelnsyndrom (Gregg-Syndrom).

Untersucht wurde außerdem die Höhe der einzelnen Antikörperwerte und ihre Verteilung auf unterschiedliche Altersklassen, hier besonders bei der Altersklasse der Neugeborenen (bis 12 Monate), der Kleinkinder, Teenager, (jungen) Erwachsenen und Personen über 50 Jahre.

\section{Ergebnisse}

Betrachtet wurden die unterschiedlichen Seroprävalenzen bei Masern, Mumps und Röteln in den einzelnen Altersgruppen. Es zeigte sich, dass bei Masern und Mumps ein etwas schnellerer Antikörperanstieg erfolgte als bei Röteln: Eine etwa 80\%ige Seropositivität wurde bei Mumps in der Altersgruppe der 5- bis 9-Jährigen erreicht, bei Masern waren hier bereits schon über $80 \%$ seropositiv und blieben es auch weiterhin in den älteren Personengruppen, während sich bei den Röteln eine $80 \%$ ige Durchseuchung erst bei den Jugendlichen finden ließ (Abb.1). Es fiel auf, dass die Seropositivitätsrate gegenüber Mumps bei jungen Erwachsenen leicht abnahm bzw. schwankte und erst bei der Gruppe der über 40-Jährigen etwa 100\% erreichte; bei Masern und Röteln wurde dagegen eine etwa 100\%ige Seroprävalenz bereits in den jüngeren Altersklassen erzielt. Im Vergleich dazu ließ sich bei der Immunitätsentwicklung für VZV bereits bei den 10- bis 14-Jährigen sehr schnell eine etwa 90\%ige Durchseuchung finden (Abb.2). Allerdings wurde ein Abfall der VZV-Seroprävalenz in der Altersgruppe der 1- bis 4Jährigen deutlich, der im Vergleich bei den Prävalenzstudien zu Masern, Mumps und Röteln in dieser Altersklasse nicht auftrat.

Bei der Immunitätslage gegenüber Röteln fanden wir in der Altersgruppe der 15- bis 19-Jährigen eine Immunitätslücke von $8,3 \%$ (8,3\% Seronegative), die dann in den folgenden Altersstufen weiter abnahm (Abb.3). Eine Aussage zur geschlechtsspezifischen Immunität war nur eingeschränkt möglich, da bei den von uns untersuchten Probanden gerade bei Röteln in den Altersstufen von 15 - 49 Jahren der Anteil der männlichen Probanden nur 4\% betrug und die Auswertungsdaten in diesem Bereich somit die Immunitätlage der weiblichen Probanden widerspiegelte. 

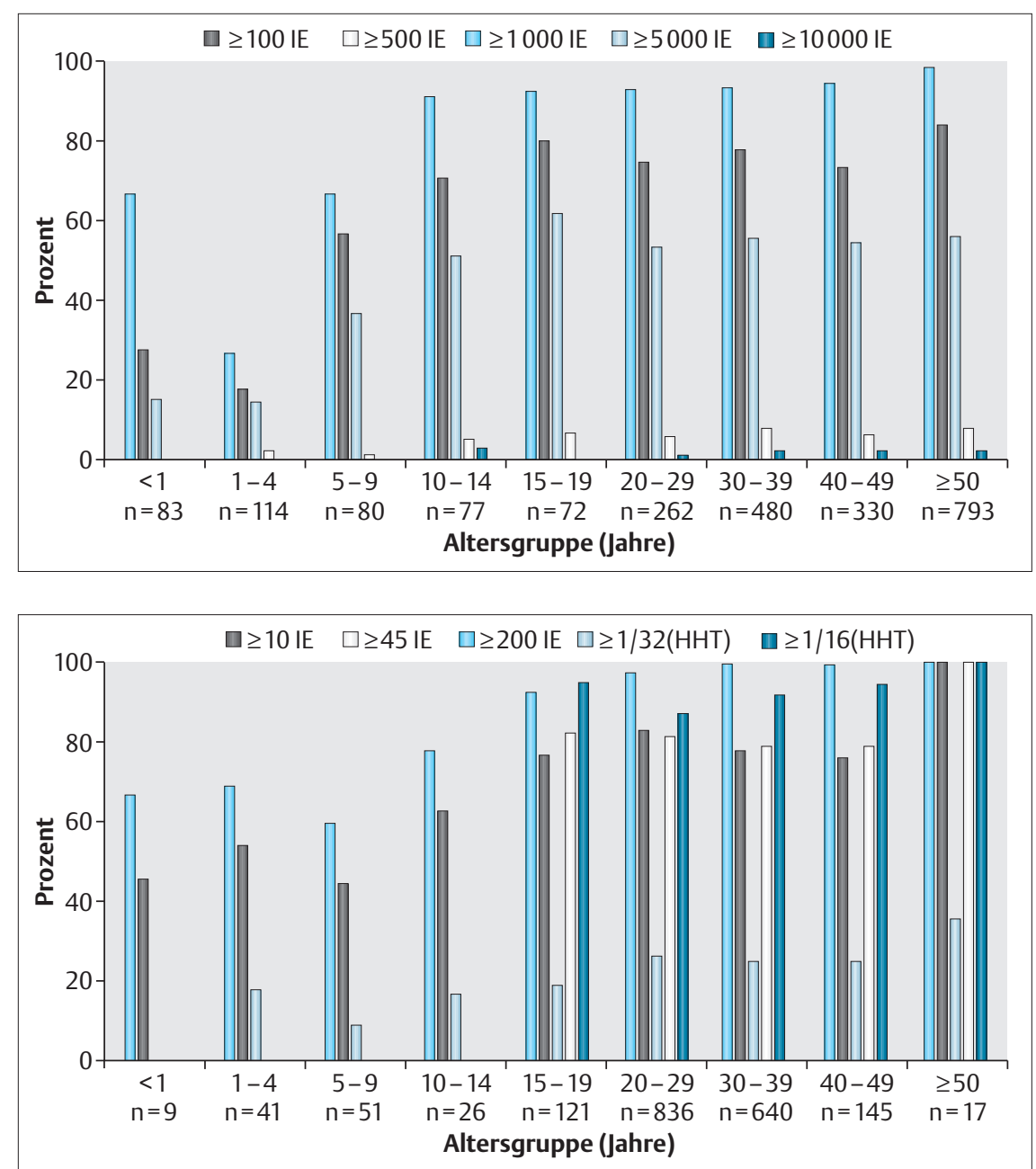

Abb. 2 Varizellen-IgG-Prävalenz in verschiedenen Altersklassen.
Abb.3 Röteln-Immunstatus in verschiedenen Altersklassen.
Hinsichtlich der Höhe der Rötelnvirus-spezifischen Antikörperwerte zeigte sich, dass erst ab der Altersstufe der 15- bis 19-Jährigen knapp $80 \%$ der Untersuchten Antikörperwerte $\geq$ $45 \mathrm{IE} / \mathrm{ml}$ aufwiesen, dies war auch in etwa der Bereich, bei dem im ELISA-Test von einer Immunität ausgegangen werden konnte. Eine sicher ausreichende Immunität (HHT-Titer $\geq$ $1: 32$ ) ließ sich in unserer Auswertung ebenfalls erst in der Altersgruppe der 15 - bis 19-Jährigen bei über $80 \%$ der Probanden nachweisen (Abb.3). Eine nahezu 100\%ige Immunität wurde bei der Personengruppe über 50 Jahre bestimmt. Auch die Anzahl der Personen mit sehr hohen Röteln-IgG-Werten $(\geq 200 \mathrm{IE} / \mathrm{ml}$ ) stieg mit dem Lebensalter an.

Beim Vergleich der Antikörperwerte von Masern, Mumps und Varizellen ab der Altersgruppe der Teenager fanden sich hohe Werte ( $\geq 1000 \mathrm{IE} / \mathrm{ml}$ ) bei weniger als $5 \%$ der auf Mumps-IgG Untersuchten, aber bei etwa 55\% der VZV-IgG Positiven. Dagegen stieg die Prozentzahl der Personen, die IgG-Werte gegenüber Masern von $\geq 1000 \mathrm{IE} / \mathrm{ml}$ aufwiesen, mit zunehmendem Lebensalter weiterhin an und erreichte letztendlich knapp 100\% (Abb.2,4 und 5).

Ergänzend zu den IgG-Antikörpern gegenüber Varizellen, waren Antikörper der Klasse IgA als Marker einer möglichen Reaktivierung bei $70 \%$ der IgG Positiven nachweisbar, signifikant erhöhte Werte ( $\geq 1280 \mathrm{IE} / \mathrm{ml}$ ) fanden sich dagegen nur bei $15 \%$.

\section{Diskussion}

Durch Bestimmung erregerspezifischer Serumantikörper lassen sich Aussagen zum Immunstatus einer Bevölkerung treffen, bei dem die Immunität durch das Durchmachen der Infektion oder durch Impfung erreicht werden kann. Von Bedeutung ist dies auch für die Eradikation einer Erkrankung, z. B. der Masern. Trotz des einfach zu führenden Nachweises spezifischer IgG-Antikörper ist die Fragestellung nach einer sicher ausreichenden Immunität gerade bei Masern, Mumps und Varizellen nicht eindeutig zu klären (fehlende internationale Standards). Lassen sich IgGAntikörper sicher nachweisen, so kann von einer Immunität ausgegangen werden. Grenzwertige Ergebnisse im ELISA-Test können Immunität bedeuten, wenn neutralisierende Antikörper im Infektionsschutzversuch vorhanden sind. Allerdings kann es gerade nach Impfung im zeitlichen Verlauf zum Abfall oder sogar Verlust der Antikörper kommen. Die Einführung von Schutzimpfungen führte zu Veränderungen der epidemiologischen Charakteristiken, wie z. B. zu einem Ausbleiben der jahreszeitlichen Schwankungen der Morbidität (Masern, Mumps), zu einer Verschiebung dieser Erkrankungen in das höhere Lebensalter, bzw. zu einer Regulierung von Epidemien $(7,8)$.

In den letzten 30 Jahren hat sich hinsichtlich der Immunitätslage kaum etwas geändert, ausgenommen dass ein Teil der Wildvirusimmunität durch die der Impfvirusinfektion ersetzt wurde $(2,14)$. 


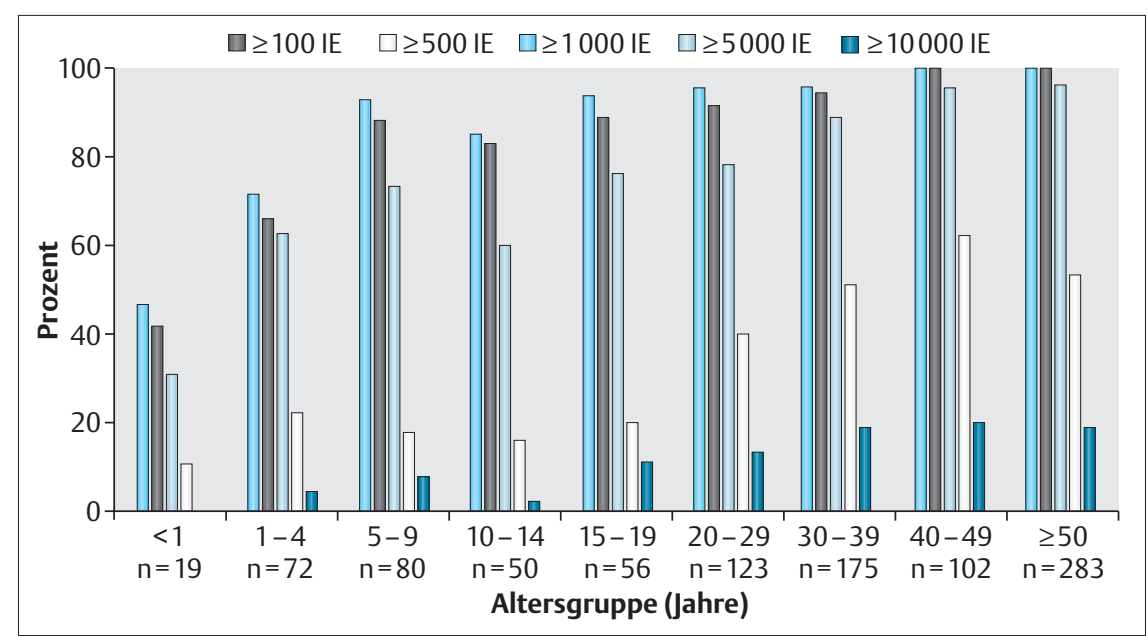

Abb.4 Masern-IgG-Prävalenz in verschiedenen Altersklassen.

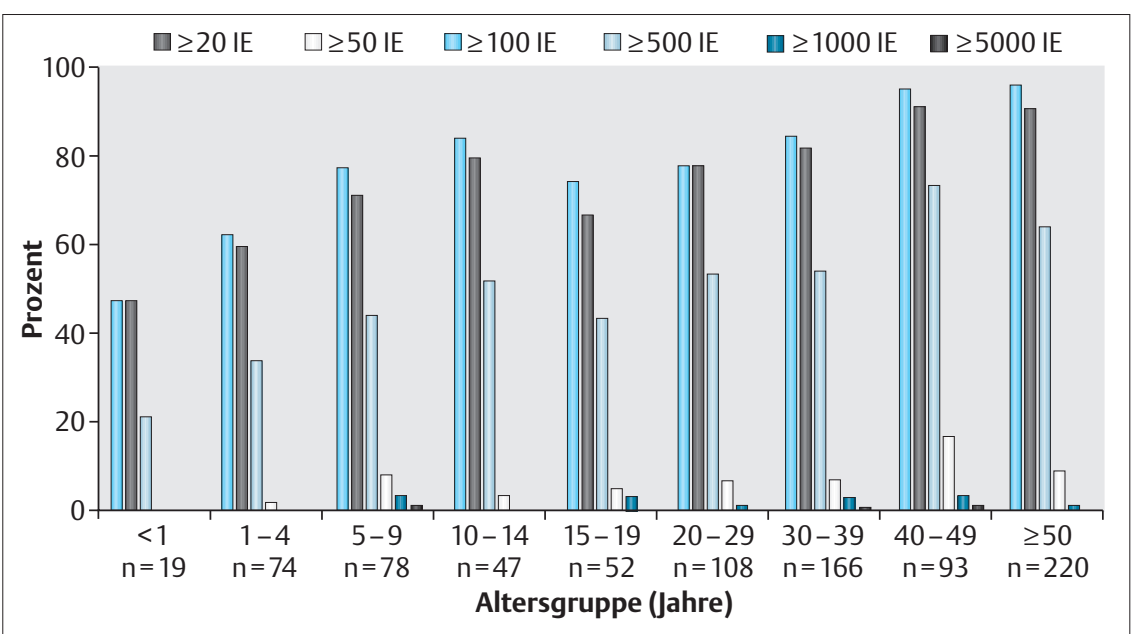

Abb.5 Mumps-IgG-Prävalenz in verschiedenen Altersklassen.

Wie auch aus anderen Untersuchungen bekannt $(2,14)$, finden sich die höchsten Immunitätsdefizite im Kindesalter. Dies konnten wir in unserer Studie bestätigen. Immunitätslücken von über $60 \%$ finden sich bei VZV in der Altersgruppe der 1bis 4-jährigen Kinder; dieser Abfall der Seroprävalenz spiegelt eindrucksvoll den Verlust maternaler Antikörper wider. Bei Masern, Mumps und Röteln liegen dagegen die Immunitätslücken bei maximal $40 \%$. Hier kommt die seit den 80er Jahren eingeführte MMR-Impfung zum Tragen, die nach den STIKOEmpfehlungen zwischen dem 12. und 15. Lebensmonat erfolgen sollte. Allerdings steigt dann die Seroprävalenz bei VZV sehr schnell an: Bereits in der Gruppe der 5- bis 9-Jährigen ist eine etwa $70 \%$ ige Immunität vorhanden - bedingt durch den hohen Kontagiositätsindex dieser Erkrankung - die dann in den älteren Jahrgängen konstant bei über $90 \%$ liegt; hier finden sich keine Veränderungen zu früheren Untersuchungen (12). Obwohl in Deutschland die Erkrankungshäufigkeiten von Masern, Mumps und Röteln stark rückläufig sind, ist eine Elimination dieser Erkrankungen noch lange nicht erreicht (15). Durch Immunisierungsraten von über $95 \%$ in den gefährdeten Jahrgängen des frühen Kindesalters würden sich diese Viruserkrankungen ausrotten lassen. Darauf zielt auch die Impfstrategie der STIKO durch die zweimalig empfohlene MMR-Impfung ab; dabei sollte die Erstimpfung zwischen 12. bis 15. Lebensmonat und die Zweitimpfung im Vorschulalter erfolgen. Auch eine Varizellen-Impfung in dieser Altersgrup- pe wäre in Betracht zu ziehen, um Ansteckungsquellen für gefährdete Personengruppen (Schwangere, Immunsupprimierte) zu verringern.

In unserer Auswertung finden wir, dass die Mehrheit der auf Röteln-Antikörper untersuchten Personen Werte von $\geq 45$ IE/ ml aufweist: Ab der Altersgruppe der 15- bis 19-Jährigen haben etwa $80 \%$ der Untersuchten sowohl Antikörperwerte von $\geq$ $45 \mathrm{IE} / \mathrm{ml}$ im ELISA-Test, als auch Titerwerte von mindestens 1:32 im Röteln-HHT, sodass von einer gesicherten Immunität ausgegangen werden kann (s. Abb.3). Trotz der MMR-Impfung zeigen sich immer noch deutliche Immunitätslücken gegenüber Röteln im Kindesalter, die jedoch auf weniger als $10 \%$ ab der Altersstufe der 15- bis 19-Jährigen zurückgehen. Übereinstimmend zu den Auswertungen von Tischer und Gericke (14) findet sich bei dieser Altersgruppe eine Immunitätslücke von $8,3 \%$, die danach auf weniger als 3,3\% abfällt. Dies verhält sich ähnlich bei den Antikörperbestimmungen gegenüber Masern und Varizellen, nur bei Mumps liegt die Immunitätslücke in dieser Altersgruppe noch bei über $20 \%$ und sinkt erst ab der Altersstufe der über 40-Jährigen unter 10\% (s. Abb.1).

Trotz abnehmender Immunitätslücken gegenüber Röteln findet sich bei den getesteten (hauptsächlich weiblichen) Personen jedoch immer ein etwa gleichbleibender Prozentsatz an Personen mit nicht sicher ausreichender Immunität im Anti- 
körper-ELISA. Gerade bei den gebärfähigen Frauen sollte dann eine Abklärung der Immunitätslage durch eine weitere Nachweismethode (z.B. HHT) erfolgen.

Unsere Auswertung zeigt, dass es bei den Mumps-Antikörpern zu einer kontinuierlichen Zunahme der Antikörperaktivitäten mit steigendem Lebensalter kommt und wir auch bei den masernspezifischen Antikörpern mit ansteigendem Lebensalter überwiegend höhere Aktivitäten als in den jüngeren Altersklassen finden. Eine Erklärung könnte zum einen die Immunisierung durch Wildvirusinfektion sein, die gerade bei den älteren Personen und damit vor Einführung der MMR-Impfung eine Rolle spielen dürfte und deren immunogene Wirkung größer ist als die durch Impfviren erzielte, desweiteren unbemerkte Boosterungen im Laufe des Lebens durch erneute Kontakte mit diesen Viren. Betrachtet man die Höhe der Varizellen-Antikörper, so lassen sich ab dem jungen Erwachsenenalter keine Anstiege der Antikörperwerte mehr finden (s. Abb.2). Deutlich erhöhte Werte bei den VZV-IgA spezifischen Antikörpern als Marker einer Varizellen-Reaktivierung konnten wir bei nur 15\% der auf VZV-IgA Untersuchten nachweisen. Dies deckt sich mit dem geschätzten Risiko für das Auftreten eines Herpes Zoster in einer Gesamtbevölkerung, das mit 10-20\% angegeben wird (16). Hypothetisch könnte man vermuten, dass das Auftreten eines Zoster mit einem Abfall VZV spezifischer IgG-Antikörpertiter einhergeht. Allerdings lässt sich in unserer Auswertung keine Abnahme der humoralen Immunität mit zunehmenden Lebensalter feststellen. Andere Faktoren bei einer Reaktivierung, hauptsächlich die Abnahme der zellulären Immunität (16), sind hier von Bedeutung.

\section{Folgerungen}

Trotz ausreichend verfügbarer Impfstoffe bestehen bei Masern, Mumps und Röteln noch große Impflücken, die am wenigsten durch "primäres" oder "sekundäres» Impfversagen (4), sondern schlicht durch Nichtimpfen auftreten. Gerade auch hinsichtlich vermeidbarer Infektionen während der Schwangerschaft, insbesondere der kongenitalen Rötelninfektion, sind die empfohlenen Impfschemata dringend einzuhalten. Die weltweite Eradikation der Masern ist angestrebtes Ziel der World Health Organization (WHO). Die Erkrankung an Masern gehört mit zu den sechs führenden Infektionskrankheiten, die etwa $90 \%$ der infektionsbedingten Todesfälle verursachen (WHO Report on Infectious Diseases, 1999). In Deutschland, wo pro Jahr noch 10000 30000 akute Masernfälle vermutet werden, kann die Erkrankung bei Erwachsenen und besonders bei Immunsupprimierten sehr schwer verlaufen.

Durch angestrebte Immunisierungsraten von über 95\% in den gefährdeten Jahrgängen ließe sich allerdings eine Ausrottung dieser impfpräventablen Viruserkrankungen erreichen. Darauf zielt auch die Impfstrategie der STIKO durch die zweimalig empfohlene Impfung zumindest gegen Masern, Mumps und Röteln ab; dabei sollte die Erstimpfung ab dem 12. bis 15. Lebensmonat und die Zweitimpfung im Vorschulalter erfolgen.

Immunitätsdefizite bei Varizellen treten hingegen nur im Kleinkindalter auf - hier besonders nach Verlust maternaler Antikörper nach dem ersten Lebensjahr. Dies wird nicht durch ein Impfprogramm ausgeglichen, da in Deutschland die Varizellenimpfung (noch) eine Indikationsimpfung darstellt.

\section{Literatur}

1 Dietz K, Schenzle D. Epidemiologische Auswirkungen von Schutzimpfungen gegen Masern, Mumps und Röteln. Marburg/Lahn: Die Medizinische Verlagsgesellschaft $\mathrm{mbH}$, In: Spiess $\mathrm{H}$ (Hrsg.): Schutzimpfungen. Notwendigkeit Wirkung/ Nebenwirkungen Impfpolitik. 1985: 219-251

2 Doerr HW, Allwinn R, Berger A. Zur Populationsimmunität gegen Masern, Mumps und Röteln 1990-1997. Epidemiologisches Bulletin des Robert Koch-Instituts 1998; 17: 122

3 Doerr HW, Braun R, Enders G. Röteln-Infektion in der Schwangerschaft. Methoden zur Risikobemessung. Münch Med Wochenschr 1983; 125: 181-184

4 Fescharek R, Quast U, Maass G, Merkle W, Schwarz S. Measlesmumps vaccination in the FRG, an empirical analysis I. Efficacy and analysis of vaccine failures. Vaccine 1990; 8: 330-336

5 Franck S, Allwinn R, Doerr HW. Zur aktuellen Poliomyelitis-Immunität. Seroepidemiologische Untersuchung auf der Basis von Patientenseren aus dem Rhein-Main-Gebiet. Epidemiologisches Bulletin des Robert Koch-Instituts 1999; 8: 49-51

6 Franck S, Allwinn R, Rabenau HF, Doerr HW. Epidemiological Analysis of immunity to Poliovirus after Termination of an Era of Vaccination with OPV in Germany. An Analysis of the German Association against Viral Diseases (DVV). Zent bl Bakteriol 1999; 289: 475-481

7 Gerike E, Tischer A. Masernvirus. Berlin/Wien: Blackwell Wissenschafts-Verlag, In: Porstmann T, Hrsg. Virusdiagnostik. 1996; 1: 327-345

8 Gerike E, Tischer A. Mumpsvirus. Berlin/Wien: Blackwell Wissenschafts-Verlag, In: Porstmann T, Hrsg. Virusdiagnostik. 1996; 1: 346-362

9 Katzmann W, Dietz K. Evaluation of age-specific vaccination strategies. Theor Popul Biol 1984; 25: 125-137(2

10 Maass G, Weber B, Doerr HW. Untersuchungen zur Immunitätslage gegen Poliomyelitis. 5. kooperative Studie der Deutschen Vereinigung zur Bekämpfung der Viruskrankheiten e.V. Dtsch Med Wochenschr 1991; 116: 1457-1462

11 Meister W, Neiss A, Gross G, Doerr HW, Höbel W, Malin J-P, von Essen J, Reimann B-Y, Witke C, Wutzler P. Demography, Symptomatology, and Course of Disease in Ambulatory Zoster Patients. A Physician-Based Survey in Germany. Intervirology 1998; 41: 272277

12 Schneweis K, Krentler C, Wolff M. Durchseuchung mit dem Varicella-Zoster-Virus und serologische Feststellung der Erstinfektionsimmunität. Dtsch Med Wochenschr 1985; 110: 453-457

13 Ständige Impfkommission am Robert Koch-Institut. Impfempfehlungen der ständigen Impfkommission (STIKO) am Robert KochInstitut. Epidemiologisches Bulletin des Robert Koch-Instituts 2001; 28: 203-218

14 Tischer A, Gerike E. Rötelnsituation in Deutschland. Bundesgesundheitsblatt 2000; 12: 940-949

15 Windorfer A. Eradikation von Masern, Mumps und Röteln. Immunologie \& Impfen, 2000; 3: 63-66

16 Wutzler P, Doerr HW. Der Herpes Zoster-ein Morbus Herpes non simplex. Dtsch Med Wochenschr 1995; 120: 1133-1138

\section{Korrespondenz}

Dr. Sigune Buxbaum

Institut für Medizinische Virologie

der Universitätsklinik Frankfurt am Main

Paul Ehrlich Straße 40

60596 Frankfurt

Tel.: 069/6301-83062

Fax: 069/6301-83061

E-Mail: S.Buxbaum@em.uni-frankfurt.de 